\title{
A Note on the Differences of Two Positive Linear Operators
}

\author{
ViJAY GUPTA AND GANCHO TACHEV
}

ABSTRACT. In the present note we find the general estimate in terms of Păltăneaś modulus of continuity. In the end, we consider some examples and we apply our result for such examples to obtain the quantitative estimates for the difference of operators.

Keywords: Weighted modulus, Baskakov operators, Szász-Mirakyan operators, Phillips operators, Lupaş operators, Difference of operators.

2010 Mathematics Subject Classification: 41A25, 41A30.

\section{INTRODUCTION}

In the last ten years there is an increasing interest to estimate the difference between two linear positive operators (abvr. 1.p.o.) in terms of appropriate moduli or $K$-functionals, see for example [4], [5], [2], [3] and [6] etc. This note is motivated by the recent paper of Aral-InoanRasa [3], where they considered two different 1.p.o. defined on unbounded interval $[0, \infty)$ and obtained estimates for the difference of these operators in a quantitative form. They defined by $C_{2}[0, \infty)$ the space of all continuous functions satisfying the condition $|f(t)| \leq M\left(1+x^{2}\right)$. Further $C_{2}^{*}[0, \infty)$ is the closed subspace of $C_{2}[0, \infty)$ formed by the functions $f$, for which $\lim _{x \rightarrow \infty}|f(x)|\left(1+x^{2}\right)^{-1}$ exists and is finite and used the norm $\|f\|_{2}=\sup _{x \geq 0}|f(x)|\left(1+x^{2}\right)^{-1}$. The weighted modulus of continuity $\Omega(f, \delta)$ (see [1]), for each $f \in C_{2}[0, \infty)$ is defined as

$$
\boldsymbol{\Omega}(f, \delta)=\sup _{|h|<\delta, x \in \mathbb{R}^{+}}|f(x+h)-f(x)|\left(1+h^{2}+x^{2}+h^{2} x^{2}\right)^{-1} .
$$

In our note we extend the class of approximated functions, including unbounded functions of polynomial growth of order $m, m \geq 2$-arbitrary natural number. We point out that the modulus $\boldsymbol{\Omega}(f,$.$) given in (1.1) is defined only for functions of polynomial growth up to order 2$. Instead of modulus $\Omega(f,$.$) , we use weighted modulus \omega_{\varphi}(f ; h)$ introduced by Pǎltănea in [10] and defined as

$$
\omega_{\varphi}(f ; h)=\sup \left\{|f(x)-f(y)|: x \geq 0, y \geq 0,|x-y| \leq h \varphi\left(\frac{x+y}{2}\right)\right\}, h \geq 0
$$

where $\varphi(x)=\frac{\sqrt{x}}{1+x^{m}}, x \in[0, \infty), m \in \mathbb{N}, m \geq 2$. We consider here those functions, for which we have the property

$$
\lim _{h \rightarrow 0} \omega_{\varphi}(f ; h)=0 .
$$

It is easy to verify that this property is fulfilled for $f$ an algebraic polynomial of degree $\leq m$. Following Theorem 2 in [10] the limit given above is true iff $f$ satisfies the following two conditions: 
- The function $f \circ e_{2}$ is uniformly continuous on $[0,1]$

- The function $f \circ e_{v}, v=\frac{2}{2 m+1}$ is uniformly continuous on $[1, \infty)$, where $e_{v}(x)=x^{v}, x \geq$ 0

We denote by $W_{\varphi}[0, \infty)$ the subspace of all real functions defined on $[0, \infty)$, satisfying above conditions. This is the first advantage of our results, compared with [3]. Secondly the authors in [3] considered the differences of two discrete l.p.o. with similar structure i.e. with the same basis functions (see (2.5) in [3])

Let $F: D \rightarrow \mathbb{R}$ be a positive linear functional, where $D$ is a linear subspace of $C[0, \infty)$ which contains $C_{2}[0, \infty)$ and the polynomials up to degree 6 and for $r \in \mathbb{N}, e_{r}(x)=x^{r}, x \in[0, \infty)$, such that $F\left(e_{0}\right)=1, b^{F}=F\left(e_{1}\right)$ and we denote $\mu_{r}^{F}=F\left(\left(e_{1}-b^{F} e_{0}\right)^{r}\right), r \in \mathbb{N}, 0 \leq r \leq 6$.

Now we consider two positive linear operators namely $M_{n}$ and $L_{n}$ for linear positive functionals $F_{n, k}, G_{n, k}: D \rightarrow \mathbb{R}$ such that $F_{n, k}\left(e_{0}\right)=1$ and $G_{n, k}\left(e_{0}\right)=1$

as

$$
M_{n}(f, x)=\sum_{k \in \mathbb{K}} F_{n, k}(f) p_{n, k}(x)
$$

and

$$
L_{n}(f, x)=\sum_{k \in \mathbb{K}} G_{n, k}(f) p_{n, k}(x)
$$

where $\mathbb{K}$ be a set of non-negative integers and the functions $p_{n, k}(x)$ are positive. One of the main results in [3] states the following:

Theorem A. Let $f \in C_{2}[0, \infty)$ with $f^{\prime \prime} \in C_{2}^{*}[0, \infty)$. Then

$$
\left|\left(M_{n}-L_{n}\right)(f, x)\right| \leq \frac{1}{2}|| f^{\prime \prime} \|_{2} \beta(x)+8 \Omega\left(f^{\prime \prime}, \delta_{1}\right)(1+\beta(x))+16 \Omega\left(f, \delta_{2}\right)(\gamma(x)+1),
$$

where

$$
\begin{aligned}
\beta(x) & =\sum_{k \in \mathbb{K}} p_{n, k}(x)\left\{\left(1+\left(b^{F_{n, k}}\right)^{2}\right) \mu_{2}^{F_{n, k}}+\left(1+\left(b^{G_{n, k}}\right)^{2}\right) \mu_{2}^{G_{n, k}}\right\}, \\
\gamma(x) & =\sum_{k \in \mathbb{K}} p_{n, k}(x)\left(1+\left(b^{F_{n, k}}\right)^{2}\right), \\
\delta_{1}^{4}(x) & =\sum_{k \in \mathbb{K}} p_{n, k}(x)\left\{\left(1+\left(b^{F_{n, k}}\right)^{2}\right) \mu_{6}^{F_{n, k}}+\left(1+\left(b^{G_{n, k}}\right)^{2}\right) \mu_{6}^{G_{n, k}}\right\}
\end{aligned}
$$

and

$$
\delta_{2}^{4}(x)=\sum_{k \in \mathbb{K}} p_{n, k}(x)\left(1+\left(b^{F_{n, k}}\right)^{2}\right)\left(b^{F_{n, k}}-b^{G_{n, k}}\right)^{4},
$$

where we suppose that $\delta_{1}(x) \leq 1, \delta_{2}(x) \leq 1$ and $\mu_{r}^{F_{n, k}}=F_{n, k}\left(\left(e_{1}-b^{F_{n, k}} e_{0}\right)^{r}\right), r \in \mathbb{N}$.

Instead of this, we study in our note the difference of two operators with different basis functions and even more $M_{n}$ and $L_{n}$ can be arbitrary positive linear operators, including integral representation. The only information we need is a good (exact if possible) representation of moments of the operators $M_{n}$ and $L_{n}$ of order upto 6 . 


\section{Auxiliary Results}

In our note we consider 1.p.o. $L_{n}: E \rightarrow C[0, \infty)$, where $E$ is a subspace of $C[0, \infty)$, such that $C_{k}[0, \infty) \subset E$, with $k=\max \{m+r+1,2 r+2,2 m\}, r \in \mathbb{N}$ and

$$
C_{k}[0, \infty):=\left\{f \in C[0, \infty), \exists M>0:|f(x)| \leq M\left(1+x^{k}\right), \forall x \geq 0, k \in \mathbb{N}\right\} .
$$

Let $\mu_{n, m}^{L}(x), m \in \mathbb{N}$ is the moment of order $m$ of $L_{n}$ i.e. $\mu_{n, m}^{L}(x)=L_{n}\left((t-x)^{m} ; x\right)$. The main result in [8] is Theorem 2.2, which we formulate as:

Theorem B. Let $L_{n}: E \rightarrow C[0, \infty), C_{k}[0, \infty) \subset E, k=\max \{m+3,6,2 m\}$ be sequence of linear positive operators, preserving the linear functions. Also $m \in \mathbb{N}$. If $f \in C^{2}[0, \infty) \cap E$ and $f^{\prime \prime} \in W_{\varphi}[0, \infty)$, then we have for $x \in(0, \infty)$ that

$$
\begin{aligned}
& \left|L_{n}(f, x)-f(x)-\frac{1}{2} f^{\prime \prime}(x) \mu_{n, 2}^{L}(x)\right| \\
\leq & \frac{1}{2}\left[\mu_{n, 2}^{L}(x)+\sqrt{2} \sqrt{L_{n}\left(\left[1+\left(x+\frac{|t-x|}{2}\right)^{m}\right]^{2} ; x\right)}\right] \\
& \omega_{\varphi}\left(f^{\prime \prime} ;\left(\frac{\mu_{n, 6}^{L}}{x}\right)^{1 / 2}\right) .
\end{aligned}
$$

Remark 2.1. We point out that the statement in Theorem B can be extended also for p.l.o. $L_{n}$ which don't preserve linear function. In this case the left hand side of (2.4) should be replaced by

$$
\left|L_{n}(f, x)-f(x)-f^{\prime}(x) \mu_{n, 1}^{L}(x)-\frac{1}{2} f^{\prime \prime}(x) \mu_{n, 2}^{L}(x)\right| .
$$

Remark 2.2. The proof of Theorem B is based on good upper estimate for the remainder in Taylor's formula at the point $x \in(0, \infty)$

$$
R_{r}(f ; t, x)=f(t)-\sum_{k=0}^{r} \frac{f^{(k)}(x)}{k !}(t-x)^{r} .
$$

From estimate (2.4) in [8], we have

$$
\left|R_{r}(f ; t, x)\right| \leq \frac{|t-x|^{r}}{r !}\left(1+\sqrt{2} \frac{|t-x|}{h} \cdot \frac{1+\left(x+\frac{|t-x|}{2}\right)^{m}}{\sqrt{x}}\right) \omega_{\varphi}\left(f^{(r)} ; h\right) .
$$

Hence for $r=0$ we get

$$
|f(t)-f(x)| \leq\left[1+\sqrt{2} \cdot \frac{|t-x|}{h} \cdot \frac{1+\left(x+\frac{|t-x|}{2}\right)^{m}}{\sqrt{x}}\right] \omega_{\varphi}(f ; h),
$$

which can be considered as an extension of the estimate (2.2) in [3], because now we allow the function $f$ to be of polynomial growth $m, m \geq 2$.. In our paper [8] we supposed that $\mu_{n, m}^{L}(x)=O\left(n^{-[(m+1) / 2]}\right), n \rightarrow$ $\infty$, which guarantees that the term in right-hand side of Theorem B in front modulus $\omega_{\varphi}\left(f^{\prime \prime},.\right)$ is bounded when $n \rightarrow \infty$ for fixed $x$ and $m$. Particularly this assumption is fulfilled for Szász-Mirakyan, Baskakov, Phillips operators etc.

Remark 2.3. We may apply also Theorem 2.3 from [8] where as argument of the modulus $\omega_{\varphi}\left(f^{\prime \prime},.\right)$ in Theorem B instead of $\left(\frac{\mu_{n, 6}^{L}}{x}\right)^{1 / 2}$ we have $\sqrt{\frac{\mu_{n, 4}^{L}(x)}{\mu_{n, 2}^{L}(x)}}$. We omit the details in this case. 


\section{MAin RESUlt}

Theorem 3.1. Let $L_{n}, M_{n}: E \rightarrow C[0, \infty), C_{k}[0, \infty) \subset E, k=\max \{m+3,6,2 m\}$ be two sequences of linear positive operators. If $f \in C^{2}[0, \infty) \cap E$ and $f^{\prime \prime} \in W_{\varphi}[0, \infty)$, then we have for $x \in(0, \infty)$ that

$$
\begin{aligned}
& \left|L_{n}(f, x)-M_{n}(f, x)\right| \\
& \leq\left|f^{\prime}(x)\right| \cdot\left|\mu_{n, 1}^{L_{n}}(x)-\mu_{n, 1}^{M_{n}}(x)\right| \\
& +\frac{1}{2}\left|f^{\prime \prime}(x)\right| \cdot\left|\mu_{n, 2}^{L_{n}}(x)-\mu_{n, 2}^{M_{n}}(x)\right| \\
& +\frac{1}{2}\left[\mu_{n, 2}^{L_{n}}(x)+\sqrt{2} \cdot \sqrt{L_{n}\left(\left[1+\left(x+\frac{|t-x|}{2}\right)^{m}\right]\right)}\right] \omega_{\varphi}\left(f^{\prime \prime} ;\left(\frac{\mu_{n, 6}^{L_{n}}}{x}\right)^{1 / 2}\right) \\
& +\frac{1}{2}\left[\mu_{n, 2}^{M_{n}}(x)+\sqrt{2} \cdot \sqrt{M_{n}\left(\left[1+\left(x+\frac{|t-x|}{2}\right)^{m}\right]\right.}\right] \omega_{\varphi}\left(f^{\prime \prime} ;\left(\frac{\mu_{n, 6}^{M_{n}}}{x}\right)^{1 / 2}\right)
\end{aligned}
$$

Proof. We use the following representation

$$
\begin{aligned}
L_{n}(f, x)-M_{n}(f, x)= & L_{n}(f, x)-f(x)-f^{\prime}(x) \mu_{n, 1}^{L_{n}}(x)-\frac{1}{2} f^{\prime \prime}(x) \mu_{n, 2}^{L_{n}}(x) \\
& -\left(M_{n}(f, x)-f(x)-f^{\prime}(x) \mu_{n, 1}^{M_{n}}(x)-\frac{1}{2} f^{\prime \prime}(x) \mu_{n, 2}^{M_{n}}(x)\right) \\
& +f^{\prime}(x)\left[\mu_{n, 1}^{L_{n}}(x)-\mu_{n, 1}^{M_{n}}(x)\right]+\frac{1}{2}\left[\mu_{n, 2}^{L_{n}}(x)-\mu_{n, 2}^{M_{n}}(x)\right] .
\end{aligned}
$$

Hence the proof follows from Theorem B.

Remark 3.4. If both the operators $L_{n}$ and $M_{n}$ reproduce linear functions, we have $\mu_{n, 1}^{L_{n}} x=\mu_{n, 1}^{M_{n}} x=0$. Therefore we can omit the summand containing $f^{\prime}(x)$.

Remark 3.5. In Theorem 3.1, we used (2.6) for $r=2$. In a similar way if we suppose $f \in C^{r}[0, \infty) \cap$ $E$ and $f^{(r)} \in W_{\varphi}[0, \infty)$ we may prove estimate for the differences $L_{n}(f, x)-M_{n}(f, x)$ in terms of $\omega_{\varphi}\left(f^{(r)} ;.\right)$.

\section{EXAMPLES}

We apply Theorem 3.1 for some classical positive linear operators, some examples are given as (see [9], [7] and references therein):

Example 4.1. The Szász-Mirakyan operators are defined as

$$
S_{n}(f, x)=\sum_{k=0}^{\infty} e^{-n x} \frac{(n x)^{k}}{k !} f\left(\frac{k}{n}\right)
$$

where $x \in[0, \infty), n \in \mathbb{N}$. The central moments of the Szász-Mirakyan operators (4.7) satisfy for $m \geq 1$ the recurrence relation:

In particular

$$
\mu_{n, m+1}^{S_{n}}(x)=\frac{x}{n}\left[\mu_{n, m}^{S_{n}}(x)\right]^{\prime}+\frac{m x}{n} \mu_{n, m-1}^{S_{n}}(x) .
$$

$$
\begin{gathered}
\mu_{n, 0}^{S_{n}}(x)=1, \mu_{n, 1}^{S_{n}}(x)=0, \mu_{n, 2}^{S_{n}}(x)=\frac{x}{n}, \mu_{n, 3}^{S_{n}}(x)=\frac{x}{n^{2}}, \\
\mu_{n, 4}^{S_{n}}(x)=\frac{x}{n^{3}}+\frac{3 x^{2}}{n^{2}}, \mu_{n, 5}^{S_{n}}(x)=\frac{x}{n^{4}}+\frac{10 x^{2}}{n^{3}}, \mu_{n, 6}^{S_{n}}(x)=\frac{x}{n^{5}}+\frac{25 x^{2}}{n^{4}}+\frac{15 x^{3}}{n^{3}} .
\end{gathered}
$$


Example 4.2. The Baskakov operators are defined as

$$
B_{n}(f ; x)=\sum_{k=0}^{\infty}\left(\begin{array}{c}
n+k-1 \\
k
\end{array}\right) \frac{x^{k}}{(1+x)^{n+k}} f\left(\frac{k}{n}\right) .
$$

The moments of the Baskakov operators are well known for $m \geq 1$ the following recurrence relation holds true:

$$
\mu_{n, m+1}^{B_{n}}(x)=\frac{x(1+x)}{n}\left[\mu_{n, m}^{B_{n}}(x)\right]^{\prime}+\frac{m x(1+x)}{n} \mu_{n, m-1}^{B_{n}}(x) .
$$

In particular

$$
\begin{aligned}
\mu_{n, 0}^{B_{n}}(x)=1, \mu_{n, 1}^{B_{n}}(x)= & 0, \mu_{n, 2}^{B_{n}}(x)=\frac{x(1+x)}{n}, \mu_{n, 3}^{B_{n}}(x)=\frac{x(1+x)(1+2 x)}{n^{2}}, \\
\mu_{n, 4}^{B_{n}}(x)= & \frac{x(1+x)}{n^{3}}+\frac{6 x^{2}(1+x)^{2}}{n^{3}}+\frac{3 x^{2}(1+x)^{2}}{n^{2}}, \\
\mu_{n, 5}^{B_{n}}(x)=\frac{x+15 x^{2}+50 x^{3}+60 x^{4}+24 x^{5}}{n^{4}}+\frac{10 x^{2}+76 x^{3}+86 x^{4}+20 x^{5}}{n^{3}}, & \frac{x+31 x^{2}+180 x^{3}+390 x^{4}+360 x^{5}+120 x^{6}}{n^{5}} \\
\mu_{n, 6}^{B_{n}}(x)= & +\frac{25 x^{2}+288 x^{3}+667 x^{4}+534 x^{5}+130 x^{6}}{n^{4}} \\
& +\frac{15 x^{3}+105 x^{4}+105 x^{5}+15 x^{6}}{n^{3}} .
\end{aligned}
$$

Example 4.3. The well known Phillips operators are defined as

$$
P_{n}(f ; x)=n \sum_{k=1}^{\infty} e^{-n x} \frac{(n x)^{k}}{k !} \int_{0}^{\infty} e^{-n t} \frac{(n t)^{k-1}}{(k-1) !} f(t) d t+e^{-n x} f(0) .
$$

The Phillips operators satisfy the following recurrence relation for central moments:

$$
\mu_{n, m+1}^{P_{n}}(x)=\frac{x}{n}\left[\left(\mu_{n, m}^{P_{n}}(x)\right)^{\prime}+2 m \mu_{n, m-1}^{P_{n}}(x)\right]+\frac{m}{n} \mu_{n, m}^{P_{n}}(x), m \geq 1
$$

and in particular, we have

$$
\begin{array}{r}
\mu_{n, 0}^{P_{n}}(x)=1, \mu_{n, 1}^{P_{n}}(x)=0, \mu_{n, 2}^{P_{n}}(x)=\frac{2 x}{n}, \\
\mu_{n, 3}^{P_{n}}(x)=\frac{6 x}{n^{2}}, \mu_{n, 4}^{P_{n}}(x)=\frac{12 x^{2}}{n^{2}}+\frac{24 x}{n^{3}} \\
\mu_{n, 5}^{P_{n}}(x)=\frac{120 x}{n^{4}}+\frac{72 x}{n^{3}}+\frac{48 x^{2}}{n^{3}}, \\
\mu_{n, 6}^{P_{n}}(x)=\frac{720 x}{n^{5}}+\frac{576 x^{2}}{n^{4}}+\frac{432 x}{n^{4}}+\frac{120 x^{3}}{n^{3}} .
\end{array}
$$

Example 4.4. The Lupaş operators are defined as

$$
U_{n}(f, x):=\sum_{k=0}^{\infty} 2^{-n x} \frac{(n x)_{k}}{k ! 2^{k}} f\left(\frac{k}{n}\right) .
$$


Some of the central moments are given below:

$$
\begin{array}{r}
\mu_{n, 0}^{U_{n}}(x)=1, \mu_{n, 1}^{U_{n}}(x)=0, \mu_{n, 2}^{U_{n}}(x)=\frac{2 x}{n}, \\
\mu_{n, 3}^{U_{n}}(x)=\frac{6 x}{n^{2}}, \mu_{n, 4}^{U_{n}}(x)=\frac{26 x+12 n x^{2}}{n^{3}}, \\
\mu_{n, 5}^{U_{n}}(x)=\frac{150 x+120 n x^{2}}{n^{4}}, \mu_{n, 6}^{U_{n}}(x)=\frac{1082 x+1140 n x^{2}+120 n^{2} x^{3}}{n^{5}} .
\end{array}
$$

Using Example 4.1 and Example 4.2, we have the following quantitative estimate as application of Theorem 3.1 for the difference of Szász-Mirakyan and Baskakov operators.

Theorem 4.2. Let $S_{n}, B_{n}: E \rightarrow C[0, \infty), C_{k}[0, \infty) \subset E, k=\max \{m+3,6,2 m\}$ be two sequences of linear positive operators. If $f \in C^{2}[0, \infty) \cap E$ and $f^{\prime \prime} \in W_{\varphi}[0, \infty)$, then we have for $x \in(0, \infty)$ that

$$
\begin{aligned}
& \left|S_{n}(f, x)-B_{n}(f, x)\right| \\
\leq & \frac{x^{2}}{2 n}\left|f^{\prime \prime}(x)\right|+\frac{1}{2}\left[\frac{x}{n}+\sqrt{2 A_{n, m, x}}\right] \omega_{\varphi}\left(f^{\prime \prime} ; \sqrt{\frac{1}{n^{5}}+\frac{25 x}{n^{4}}+\frac{15 x^{2}}{n^{3}}}\right) \\
& +\frac{1}{2}\left[\frac{x(1+x)}{n}+\sqrt{2 B_{n, m, x}}\right] \omega_{\varphi}\left(f^{\prime \prime} ;\left(\frac{1+31 x+180 x^{2}+390 x^{3}+360 x^{4}+120 x^{5}}{n^{5}}\right.\right. \\
+ & \left.\left.\frac{25 x+288 x^{2}+667 x^{3}+534 x^{4}+130 x^{5}}{n^{4}}+\frac{15 x^{2}+105 x^{3}+105 x^{4}+15 x^{5}}{n^{3}}\right)^{1 / 2}\right),
\end{aligned}
$$

where

$$
A_{n, m, x}=S_{n}\left(\left[1+\left(x+\frac{|t-x|}{2}\right)^{m}\right]^{2} ; x\right), B_{n, m, x}=B_{n}\left(\left[1+\left(x+\frac{|t-x|}{2}\right)^{m}\right]^{2} ; x\right) .
$$

Using Example 4.1 and Example 4.3, we have the following quantitative estimate as application of Theorem 3.1 for the difference of Szász-Mirakyan and Phillips operators.

Theorem 4.3. Let $S_{n}, P_{n}: E \rightarrow C[0, \infty), C_{k}[0, \infty) \subset E, k=\max \{m+3,6,2 m\}$ be two sequences of linear positive operators. If $f \in C^{2}[0, \infty) \cap E$ and $f^{\prime \prime} \in W_{\varphi}[0, \infty)$, then we have for $x \in(0, \infty)$ that

$$
\begin{aligned}
& \left|S_{n}(f, x)-P_{n}(f, x)\right| \\
\leq & \frac{x}{2 n}\left|f^{\prime \prime}(x)\right|+\frac{1}{2}\left[\frac{x}{n}+\sqrt{2 A_{n, m, x}}\right] \omega_{\varphi}\left(f^{\prime \prime} ; \sqrt{\frac{1}{n^{5}}+\frac{25 x}{n^{4}}+\frac{15 x^{2}}{n^{3}}}\right) \\
+ & \frac{1}{2}\left[\frac{2 x}{n}+\sqrt{2 C_{n, m, x}}\right] \omega_{\varphi}\left(f^{\prime \prime} ; \sqrt{\frac{720}{n^{5}}+\frac{576 x}{n^{4}}+\frac{432}{n^{4}}+\frac{120 x^{2}}{n^{3}}}\right),
\end{aligned}
$$

where

$$
A_{n, m, x}=S_{n}\left(\left[1+\left(x+\frac{|t-x|}{2}\right)^{m}\right]^{2} ; x\right), C_{n, m, x}=P_{n}\left(\left[1+\left(x+\frac{|t-x|}{2}\right)^{m}\right]^{2} ; x\right) .
$$

Using Example 4.3 and Example 4.4, we have the following quantitative estimate as application of Theorem 3.1 for the difference of Phillips and Lupaş operators. 
Theorem 4.4. Let $P_{n}, U_{n}: E \rightarrow C[0, \infty), C_{k}[0, \infty) \subset E, k=\max \{m+3,6,2 m\}$ be two sequences of linear positive operators. If $f \in C^{2}[0, \infty) \cap E$ and $f^{\prime \prime} \in W_{\varphi}[0, \infty)$, then we have for $x \in(0, \infty)$ that

$$
\begin{aligned}
& \left|P_{n}(f, x)-U_{n}(f, x)\right| \\
\leq & \frac{1}{2}\left[\frac{2 x}{n}+\sqrt{2 C_{n, m, x}}\right] \omega_{\varphi}\left(f^{\prime \prime} ; \sqrt{\frac{720}{n^{5}}+\frac{576 x}{n^{4}}+\frac{432}{n^{4}}+\frac{120 x^{2}}{n^{3}}}\right) \\
+ & \frac{1}{2}\left[\frac{2 x}{n}+\sqrt{2 D_{n, m, x}}\right] \omega_{\varphi}\left(f^{\prime \prime} ; \sqrt{\frac{1082+1140 n x+120 n^{2} x^{2}}{n^{5}}}\right),
\end{aligned}
$$

where

$$
D_{n, m, x}=U_{n}\left(\left[1+\left(x+\frac{|t-x|}{2}\right)^{m}\right]^{2} ; x\right), C_{n, m, x}=P_{n}\left(\left[1+\left(x+\frac{|t-x|}{2}\right)^{m}\right]^{2} ; x\right) .
$$

\title{
REFERENCES
}

[1] T. Acar, A. Aral and I. Raşa: The new forms of Voronovskaja's theorem in weighted spaces, Positivity 20 (2016), 25-40.

[2] A. M. Acu and I. Raşa: New estimates for differences of positive linear operators, Numer. Algor. 73 (2016), 775-789.

[3] A. Aral, D. Inoan and I. Raşa: On differences of linear positive operators, Anal. Math. Phys. doi 13324-018-0227-7.

[4] H. Gonska, P. Pitul and I. Raşa: On differences of positive linear operators, Carpathian J. Math. 22(1-2) (2006), 65-78.

[5] H. Gonska, and I. Raşa: Differences of positive linear operators and the second order modulus, Carpathian J. Math. 24(3) (2008), 332-340.

[6] V. Gupta: Differences of operators of Lupaş type, Constructive Mathematical Analysis 1(1) (2018), 9-14.

[7] V. Gupta, T. M. Rassias, P. N. Agrawal and A. M. Acu: Recent Advances in Constructive Approximation Theory, Springer, Cham. (2018).

[8] V. Gupta and G. Tachev: General form of Voronovskaja's theorem in terms of weighted modulus of continuity, Results Math 69 (3-4) (2016), 419-430.

[9] V. Gupta and G. Tachev: Approximation with Positive Linear Operators and Linear Combinations, Springer, Cham. 2017.

[10] R. Păltănea: Estimates of approximation in terms of a weighted modulus of continuity, Bull. Transilvania Univ. of Brasov 4 (53) (2011), 67-74.

\author{
Netaji SubHas University of TECHNOLOGy \\ DEPARTMENT OF MATHEMATICS \\ SECTOR 3 DWARKA, NEW DELHI 110078, INDIA \\ E-mail address: vi jaygupta2001@hotmail.com \\ University of ARCHITECTURE, CIVIL ENGINEERING AND GEODESY \\ DEPARTMENT OF MATHEMATICS \\ BG-1046, SOFIA, BULGARIA \\ E-mail address: gtt_fte@uacg.acad.bg
}

